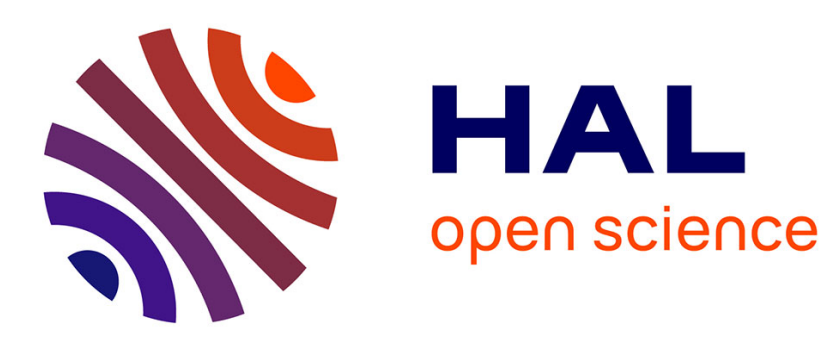

\title{
Recovering the initial state of an infinite-dimensional system using observers
}

Karim Ramdani, Marius Tucsnak, George Weiss

\section{To cite this version:}

Karim Ramdani, Marius Tucsnak, George Weiss. Recovering the initial state of an infinite-dimensional system using observers. Automatica, 2010, 46 (10), pp.1616-1625. 10.1016/j.automatica.2010.06.032 . hal-00529834

\section{HAL Id: hal-00529834 \\ https://hal.science/hal-00529834}

Submitted on 24 Feb 2013

HAL is a multi-disciplinary open access archive for the deposit and dissemination of scientific research documents, whether they are published or not. The documents may come from teaching and research institutions in France or abroad, or from public or private research centers.
L'archive ouverte pluridisciplinaire HAL, est destinée au dépôt et à la diffusion de documents scientifiques de niveau recherche, publiés ou non, émanant des établissements d'enseignement et de recherche français ou étrangers, des laboratoires publics ou privés. 


\title{
Recovering the initial state of an infinite-dimensional system using observers
}

\author{
Karim Ramdani \\ INRIA Nancy Grand-Est \\ University of Nancy \\ Vandoeuvre les Nancy 54506 \\ France \\ Karim.Ramdani@inria.fr
}

\author{
Marius Tucsnak \\ Department of Mathematics \\ University of Nancy \\ Vandoeuvre les Nancy 54506 \\ France \\ Marius.Tucsnak@iecn.u-nancy.fr
}

George Weiss

Department of EE-Systems

Tel Aviv University

Ramat Aviv 69978

Israel

gweiss@eng.tau.ac.il

\begin{abstract}
Let $A$ be the generator of a strongly continuous semigroup $\mathbb{T}$ on the Hilbert space $X$, and let $C$ be a linear operator from $\mathcal{D}(A)$ to another Hilbert space $Y$ (possibly unbounded with respect to $X$, not necessarily admissible). We consider the problem of estimating the initial state $z_{0} \in \mathcal{D}(A)$ (with respect to the norm of $X$ ) from the output function $y(t)=C \mathbb{T}_{t} z_{0}$, given for all $t$ in a bounded interval $[0, \tau]$. We introduce the concepts of estimatability and backward estimatability for $(A, C)$ (in a more general way than currently available in the literature), we introduce forward and backward observers, and we provide an iterative algorithm for estimating $z_{0}$ from $y$. This algorithm generalizes various algorithms proposed recently for specific classes of systems and it is an attractive alternative to methods based on inverting the Gramian. Our results lead also to a very general formulation of Russell's principle, i.e., estimatability and backward estimatability imply exact observability. This general formulation of the principle does not require $\mathbb{T}$ to be invertible. We illustrate our estimation algorithms on systems described by wave and Schrödinger equations, and we provide results from numerical simulations.
\end{abstract}

Keywords: strongly continuous semigroup, estimatability, exact observability, Russell's principle, back and forth nudging, time reversal focusing, wave equation.

\section{Introduction}

In many areas of science and engineering it is important to estimate the initial (or the final) state of a linear distributed parameter system (DPS) from its input and output functions measured over some finite time interval. In oceanography and meteorology this problem is called data assimilation, see for example Auroux and Blum [3, 4], Le Dimet et al [24], Teng et al [34] or Zou et al [40]. Such a problem also arises in the context of medical imaging by impedance-acoustic tomography; see for instance Gebauer and Scherzer [14] and the review paper by Kuchment and Kunyansky [22]. The estimation of the initial 
state can also be regarded as the main step in solving inverse source problems, see Alvez et al [1]. An infinite-dimensional system is called exactly observable in time $\tau$ if the problem of estimating the initial state from data measured over a time interval of length $\tau$ is well-posed (see Section 2 for precise definitions).

Suppose now that we have an exactly observable linear DPS. The actual formula for expressing the initial state from the measured segment of the input and output functions involves inverting the Gramian operator of the system (see, for instance, Tucsnak and Weiss [35, Section 6.1]), and this may be numerically very challenging.

On the other hand, if we find a stabilizing output injection operator for the system (its existence follows from exact observability), then we can use a numerical solver to implement an observer for the DPS in order to estimate its current state from the measured inputs and outputs of the system (see Section 2 for details). The estimate improves as time goes by, more precisely, the estimation error tends to zero as time goes to infinity. From the final state we can, in principle, recover the initial state if the dynamics are time reversible. However, this may increase the estimation error and moreover, sometimes we do not have a very long (in time) segment of output data to operate on.

The purpose of this paper is to describe a way in which we can estimate the initial state of a linear DPS by operating only on a finite segment of output data. In short, the idea is to scan the same segment of data back and forth several times, using two observers, one working in forward time, and one in backward time. This idea has appeared in the recent papers $[3,4]$ where the method has been mathematically justified for finitedimensional linear systems with full observation and it has been numerically investigated for more general situations (in particular nonlinear systems). A related work is Phung and Zhang [26] where, based on time reversal methods (see e.g. Fink et al. [13]), the authors develop a method to identify the initial state for a Kirchhoff plate equations with distributed observation. The algorithm in this last paper can be shown to be equivalent to a particular case of the algorithm presented in this paper (see Remark 3.11 below). In $[3,4]$ the algorithm has been called "back and forth nudging" whereas in [26] it has been called "time reversal focusing". An abstract formulation of a related algorithm, suitable for skew-adjoint generators and bounded observation operators, has been given in Ito, Ramdani and Tucsnak [17].

Linear DPS often have unbounded control and/or observation operators. This is often the consequence of boundary control and/or boundary observation (see [35] for an elementary introduction to this topic). To make our basic ideas more easily understandable, we give in this introductory section a short presentation of the simple particular case when the observation operator is bounded, the output injection operators are also bounded and the semigroup is invertible (i.e., the observed system is time reversible). However, we emphasize that our results do not require the observation and output injection operators to be bounded (not even admissible), and we also do not require the observed system to be time reversible.

Let $X$ and $Y$ be Hilbert spaces, called the state space and output space, respectively. Let $A: \mathcal{D}(A) \rightarrow X$ be the generator of a strongly continuous group of operators on $X$. This group describes the (time reversible) dynamics of our system. The system is described by the equations

$$
\dot{z}(t)=A z(t), \quad y(t)=C z(t),
$$


with an initial state $z(0) \in X$, where $y$ is the output function. We assume (in this simplified presentation) that $C \in \mathcal{L}(X, Y)$. The operator $C$ is called the observation operator. We assume that $(A, C)$ is estimatable with bounded injection, which means that there exists an $H \in \mathcal{L}(Y, X)$ such that $A+H C$ generates an exponentially stable semigroup on $X$. In this case, we can construct an observer for our system as follows: the state of the observer is $w$ and it satisfies the differential equation

$$
\dot{w}(t)=(A+H C) w(t)-H y(t),
$$

with an initial state $w(0) \in X$. We refer to Curtain and Zwart [11] for a discussion of observers in this context. If we define the estimation error by $e(t)=w(t)-z(t)$, then it is clear (from subtracting the two differential equations) that

$$
\dot{e}(t)=(A+H C) e(t),
$$

which shows that $e \rightarrow 0$ exponentially, regardless of the initial states of the system and the observer.

Now suppose that $(A, C)$ is backward estimatable with bounded injection, which means there exists an $H_{b} \in \mathcal{L}(Y, X)$ such that $-A+H_{b} C$ is the generator of an exponentially stable semigroup on $X$. In this case, we can construct a backward observer for our system as follows: the state of the observer is $w_{b}$ and it satisfies the differential equation

$$
\dot{w}_{b}(t)=\left(A-H_{b} C\right) w_{b}(t)+H_{b} y(t),
$$

with a final state $w_{b}(\tau) \in X$. This equation should be solved backward in time on $[0, \tau]$, starting from the final state $w_{b}(\tau)$. If we define the backward estimation error by $e_{b}(t)=$ $w_{b}(t)-z(t)$, then from subtracting the differential equations we see that

$$
\dot{e}_{b}(t)=\left(A-H_{b} C\right) e_{b}(t),
$$

which shows that

$$
e_{b}(0)=e^{\left(-A+H_{b} C\right) \tau} e_{b}(\tau) .
$$

We define one estimation cycle as follows: with the data $y(t)$ given for $t \in[0, \tau]$, we choose an initial state $w(0)$ for the observer and run it over the interval $[0, \tau]$, obtaining an estimate $w(\tau)$ for the unknown state $z(\tau)$. Putting $w_{b}(\tau)=w(\tau)$, we run the backward observer to obtain an estimate $w_{b}(0)$ for the unknown state $z(0)$. The estimation error at the end of such a cycle is

$$
e_{b}(0)=e^{\left(-A+H_{b} C\right) \tau} e^{(A+H C) \tau} e(0)
$$

If we run $N$ estimation cycles $(N \in \mathbb{N})$ then the estimation error at the end will be

$$
e_{b}^{(N)}(0)=\left[e^{\left(-A+H_{b} C\right) \tau} e^{(A+H C) \tau}\right]^{N} e^{(0)}(0) .
$$

Since the semigroups generated by $A+H C$ and $-A+H_{b} C$ are exponentially stable, for $\tau>0$ large enough, we have

$$
\left\|e^{\left(-A+H_{b} C\right) \tau} e^{(A+H C) \tau}\right\|<1
$$

For such $\tau$, repeating estimation cycles leads to a rapid convergence of the estimation error to zero. 
Notice that it follows from the above argument that under the stated assumptions (all the operators apart from $A$ are bounded, estimatability and backward estimatability), the original system is exactly observable. This is a dual version of Russell's principle, which was originally stated in Russell $[29,30]$ and has been used in many references, such as Komornik [18]. A rigorous and general statement and proof of the principle can be found in the little known conference paper Rebarber and Weiss [27]. The precise statement of a more general version of the dual Russell's principle (not confined to bounded operators and not requiring that the semigroup is invertible) will be given in Proposition 3.3. An even more general version will be given in Remark 3.5.

The paper is organized as follows: in Section 2, we recall some basic facts about admissibility, observability and observers. The iterative reconstruction algorithm is described in Section 3 in a general setting and then applied in Section 4 to a particular class of non reversible second order systems with unbounded observation. Finally, Section 5 provides 2D numerical examples illustratating the efficiency of the proposed reconstruction method.

\section{Background on admissibility, observability and observers}

First we give some technical background about linear DPS. More precisely, we recall some simple facts about admissibile observation and control operators, exact observability and estimatability. There is a large literature on admissibility and we refer to Chapter 5 of [35] for an elementary introduction and for references and historical comments. Here we keep the discussion to the minimum that is needed. The concept of estimatability is one of the infinite-dimensional generalizations of the well-known concept of detectability used in finite-dimensional control theory. It is much less well-known than admissibility, and our basic reference for this concept is Weiss and Rebarber [38].

Let $X$ and $Y$ be Hilbert spaces and assume that $A: \mathcal{D}(A) \rightarrow X$ is the generator of a strongly continuous semigroup $\mathbb{T}$ on $X$. We define the Hilbert space $X_{1}=\mathcal{D}(A)$, with the norm

$$
\left\|z_{0}\right\|_{1}=\left\|(\beta I-A) z_{0}\right\| \quad \forall z_{0} \in \mathcal{D}(A),
$$

where $\beta$ is an arbitrary fixed element of the resolvent set $\rho(A)$. Regardless of the choice of $\beta$, the above norm is equivalent to the graph norm.

Let $C \in \mathcal{L}\left(X_{1}, Y\right)$. For every $\tau>0$ we define the operator $\Psi_{\tau}: X_{1} \rightarrow L^{2}([0, \tau] ; Y)$ by

$$
\left(\Psi_{\tau} z_{0}\right)(t)=C \mathbb{T}_{t} z_{0} \quad \forall t \in[0, \tau], z_{0} \in \mathcal{D}(A) .
$$

We call $C$ an admissible observation operator for $\mathbb{T}$ if for some (hence, for every) $\tau>0$, $\Psi_{\tau}$ has a continuous extension to $X$, i.e., $\Psi_{\tau} \in \mathcal{L}\left(X, L^{2}([0, \tau] ; Y)\right)$. If this is the case, then there exists a unique continuous operator $\Psi: X \rightarrow L_{l o c}^{2}([0, \infty) ; Y)$ such that for every $\tau>0$ and $z_{0} \in X, \Psi_{\tau} z_{0}$ is the restriction of $\Psi z_{0}$ to the interval $[0, \tau]$. The Laplace transform of $y=\Psi z_{0}$ is $\hat{y}=C(s I-A)^{-1} z_{0}$, for all $z_{0} \in X$ and for all $s \in \mathbb{C}$ for which Re $s$ is larger than the growth bound of $\mathbb{T}$.

With $X, A$ as above and $C \in \mathcal{L}\left(X_{1}, Y\right)$, the pair $(A, C)$ is called exactly observable in time $\tau>0$ if there exists $k_{\tau}>0$ such that

$$
\int_{0}^{\tau}\left\|C \mathbb{T}_{t} z_{0}\right\|^{2} \mathrm{~d} t \geqslant k_{\tau}^{2}\left\|z_{0}\right\|^{2} \quad \forall z_{0} \in \mathcal{D}(A) .
$$


The pair $(A, C)$ is called exactly observable if it is exactly observable in some time $\tau>0$.

Note that in the above definition we have not assumed that $C$ is admissible. This is different from the terminology adopted in [35], where admissibility is a part of the definition of exact observability (in time $\tau$ ).

Let $X_{-1}$ be the completion of $X$ with respect to the norm

$$
\left\|z_{0}\right\|_{-1}=\left\|(\beta I-A)^{-1} z_{0}\right\| \quad \forall z_{0} \in X,
$$

where $\beta$ is as before. This space may be regarded as the dual of $\mathcal{D}\left(A^{*}\right)$ with graph norm, with respect to the pivot space $X$. We have $X_{1} \subset X \subset X_{-1}$, densely and with continuous embeddings. The semigroup $\mathbb{T}$ can be extended to a strongly continuous semigroup acting on $X_{-1}$, whose generator is an extension of $A$, having the domain $X$. Notationally, we shall not distinguish between $\mathbb{T}$ and its extension, or between $A$ and its extension. Let $U$ be another Hilbert space and let $B \in \mathcal{L}\left(U, X_{-1}\right)$. For every $\tau>0$ we define the operator $\Phi_{\tau}: L^{2}([0, \tau] ; U) \rightarrow X_{-1}$ by

$$
\Phi_{\tau} u=\int_{0}^{\tau} \mathbb{T}_{t-\sigma} B u(\sigma) \mathrm{d} \sigma
$$

We call $B$ an admissible control operator for $\mathbb{T}$ if for some (hence, for every) $\tau>0$, the range of $\Phi_{\tau}$ is contained in $X$. If this is the case, then $\Phi_{\tau}$ is bounded from $L^{2}([0, \tau] ; U)$ to $X$. If $u \in L_{l o c}^{2}([0, \infty) ; U)$ then by $\Phi_{\tau} u$ we mean $\Phi_{\tau}$ applied to the truncation of $u$ to $[0, \tau]$. If $B$ is admissible then $\Phi_{\tau} u$ is a continuous $X$-valued function. Moreover, for any $u \in L_{l o c}^{2}([0, \infty) ; U)$ and any $z_{0} \in X$, the function $z(t)=\mathbb{T}_{t} z_{0}+\Phi_{t} u$ satisfies $\dot{z}(t)=A z(t)+B u(t)$ in the sense that it satisfies (for all $\tau \geqslant 0$ ) the integral equation

$$
z(\tau)-z(0)=\int_{0}^{\tau}[A z(t)+B u(t)] \mathrm{d} t .
$$

In fact, the function $z$ defined earlier is the unique solution of the above integral equation that satisfies the initial condition $z(0)=z_{0}$.

Definition 2.1. Let $A$ be the generator of a strongly continuous semigroup $\mathbb{T}$ on $X$ and let $C \in \mathcal{L}\left(X_{1}, Y\right)$. The pair $(A, C)$ is estimatable if the following conditions hold:

(1) There exists an operator $A^{K}: \mathcal{D}\left(A^{K}\right) \rightarrow X$ that generates an exponentially stable semigroup $\mathbb{T}^{K}$ on $X$. We denote by $X_{-1}^{K}$ the analogue of the space $X_{-1}$ discussed earlier, for the operator $A^{K}$.

(2) There exists $H \in \mathcal{L}\left(Y, X_{-1}^{K}\right)$ that is an admissible control operator for $\mathbb{T}^{K}$, such that

$$
A x=A^{K} x-H C x \quad \forall x \in \mathcal{D}(A) .
$$

Following the finite-dimensional terminology, we call $H$ as above a stabilizing output injection operator for $(A, C)$.

Note that in (2.2), both $A^{K} x$ and $H C x$ are in $X_{-1}^{K}$, but their difference is in $X$. The equation $(2.2)$ can be rewritten equivalently in weak form as follows:

$$
\langle A x, \phi\rangle_{X}=\left\langle x,\left(A^{K}\right)^{*} \phi\right\rangle_{X}-\left\langle C x, H^{*} \phi\right\rangle_{Y} \quad \forall x \in \mathcal{D}(A), \phi \in \mathcal{D}\left(\left(A^{K}\right)^{*}\right) .
$$


Remark 2.2. In the finite-dimensional control literature as well as in the literature on DPS with bounded control and observation operators, the property defined above is called detectability. When dealing with unbounded operators, detectability has several (nonequivalent) generalizations. A more restrictive one has been given in Weiss and Curtain [37], where there are additional assumptions, for example, $(A, H, C)$ has to be a regular triple. This more restrictive concept is called detectability in [37]. The above definition of estimatability is close to, but not equal to the one in [38]. Indeed, in [38] $C$ is assumed to be admissible for $\mathbb{T}$, while here we have dropped this requirement. Even for admissible $C$, the definition of estimatability in [38] looks different from the one given here, but they are equivalent, see Propositions 3.3, 3.4 and 4.4 in [38].

Remark 2.3. It is known (see [38, Section 1]) that if $C$ is admissible for $\mathbb{T}$, then the exact observability of the pair $(A, C)$ implies its estimatability.

Remark 2.4. Given $A$ and $C$ it is usually not a simple task to find a stabilizing output injection operator $H$ for them. Some approaches involve Riccati equations (see, for instance, Lasiecka [23]), other approaches involve Gramians (see, for example, Komornik [19] and Urquiza [36]). These methods for determining $H$ are computationally expensive in general. If $A$ is skew-adjoint (or close to skew-adjoint) then often we can choose $H=-C^{*}$. Such an $H$ is called colocated output injection, and it is dual to colocated state feedback. Colocated state feedback has been studied a lot in the literature on distributed parameter systems. Among the early papers we mention Slemrod [31] and Haraux [16]. For the case of skew-adjoint $A$ and bounded $C$ see our Proposition 3.7. Curtain and Weiss [10] contains many further references on this topic and it gives rather general sufficient conditions on $(A, B)$ for $-B^{*}$ to be a stabilizing state feedback operator. Ammari and Tucsnak [2] considered the case of second order systems without damping.

The above definition of estimatability is clearly a generalization of the one given in the Introduction for bounded operators $C$ and $H$. It may seem very abstract, but it implies the solvability of a reasonably natural final state estimation problem:

Proposition 2.5. Assume that the pair $(A, C)$ is estimatable and let $\mathbb{T}, A^{K}, \mathbb{T}^{K}, H$ be as in Definition 2.1. Take $z_{0} \in \mathcal{D}(A)$, let $z \in C\left([0, \infty) ; X_{1}\right)$ be defined by $z(t)=\mathbb{T}_{t} z_{0}$ and let $y \in C([0, \infty) ; Y)$ be defined by $y=C z$. Let $w_{0} \in X$ and let $w \in C([0, \infty) ; X)$ be the solution of

$$
\dot{w}(t)=A^{K} w(t)-H y(t), \quad w(0)=w_{0},
$$

in the sense of (2.1). Then

$$
w(t)-z(t)=\mathbb{T}_{t}^{K}\left(w_{0}-z_{0}\right) \quad \forall t \geqslant 0 .
$$

In particular, there exist $M \geqslant 1, \omega>0$ such that

$$
\|w(t)-z(t)\| \leqslant M e^{-\omega t}\left\|w_{0}-z_{0}\right\| \quad \forall t \geqslant 0 .
$$

Proof. Clearly for all $t \geqslant 0$,

$$
z(t)-z_{0}=\int_{0}^{t} A z(\xi) \mathrm{d} \xi
$$

where $z$ and $A z$ are continuous $X$-valued functions. The fact that $w \in C([0, \infty) ; X)$ satisfies (2.4) implies (see (2.1)) that for all $t \geqslant 0$, 


$$
w(t)-w_{0}=\int_{0}^{t} A^{K} w(\xi) \mathrm{d} \xi-\int_{0}^{t} H y(\xi) \mathrm{d} \xi .
$$

In the above formula the two terms in the right-hand side lie in $C\left([0, \infty), X_{-1}^{K}\right)$ but their sum is in $C([0, \infty), X)$. For $t \geqslant 0$ we set $e(t)=w(t)-z(t)$. We apply both equations (2.6) and (2.7) to an arbitrary element $\phi \in \mathcal{D}\left(\left(A^{K}\right)^{*}\right)$. Then we subtract side by side the resulting equations and use (2.3), to obtain

$$
e(t)-e(0)=\int_{0}^{t} A^{K} e(\xi) \mathrm{d} \xi \quad \forall t \geqslant 0 .
$$

This implies that $e(t)=\mathbb{T}_{t}^{K} e(0)$, which is exactly (2.5). The last statement in the proposition follows now from the exponential stability of $\mathbb{T}^{K}$.

The system (2.4) is called an observer for $(A, C)$ (sometimes called a Luenberger observer or a Kalman observer). Such observers for systems with skew-adjoint $A$, unbounded $C$ and such that $\left(A, C^{*}, C\right)$ determine a regular linear system, were recently discussed in Deguenon, Sallet and Xu [12]. Other recent papers on the use of observers for the control of linear DPS are Krstic et al. [20] and Guo and Shao [15]. Observers for non-linear systems are studied in Bonnabel et al. [7] (in the finite dimensional context), Smyshlyaev and Krstic [32] and Krstic et al. [21].

Remark 2.6. Sometimes we have to estimate the initial state of a system that receives an input signal, i.e., it is described by

$$
\left\{\begin{array}{l}
\dot{z}(t)=A z(t)+B u(t), \\
y(t)=C z(t)+D u(t), \\
z(0)=z_{0} \in \mathcal{D}(A)
\end{array}\right.
$$

Here we assume that $B \in \mathcal{L}(U, X)$ and $D \in \mathcal{L}(U, Y)$, where $U$ is another Hilbert space, while $C \in \mathcal{L}\left(X_{1}, Y\right)$, as before. It is not obvious how to make sense of these equations, because $C$ is only defined on $X_{1}=\mathcal{D}(A)$. We assume, for the moment, that $u \in \mathcal{H}_{\text {loc }}^{1}((0, \infty) ; U)$. Then it follows from [35, Theorem 4.1.6] that the first equation in (2.8) has a unique classical solution $z$ and this is a continuous $X_{1}$-valued function of the time $t$. Thus, the second equation in (2.8) defines a continuous $Y$-valued function $y$. We assume that the signal $u$ is available to the observer, defined by

$$
\left\{\begin{array}{l}
\dot{w}(t)=A^{K} w(t)+(B+H D) u(t)-H y(t), \\
w(0)=w_{0} \in X .
\end{array}\right.
$$

This equation has solutions that are continuous with values in $X$, because $H$ is an admissible control operator for $\mathbb{T}^{K}$. A short computation and proof along the same lines as the proof of Proposition 2.5 shows that the error satisfies the same equation (2.5) as before. This shows that the map $\left(z_{0}, w_{0}, u\right) \mapsto w(\tau)$ (which is obtained by combining the observer with the system) can be extended by continuity to a bounded operator from $X \times X \times L^{2}([0, \tau] ; U)$ to $X$ (without assuming $C$ to be admissible).

\section{Iterations using forward and backward observers}

In order to estimate the initial state of a system iteratively using output data from a finite time interval, we need the notion of backward estimatability, defined below. In this section (as in the previous one) $X$ and $Y$ denote Hilbert spaces. 
Definition 3.1. Let $A$ be the generator of a strongly continuous semigroup $\mathbb{T}$ on $X$ and let $C \in \mathcal{L}\left(X_{1}, Y\right)$. The pair $(A, C)$ is backward estimatable if the following conditions hold:

(1) There exists an operator $A_{b}^{K}: \mathcal{D}\left(A_{b}^{K}\right) \rightarrow X$ that generates an exponentially stable semigroup $\mathbb{S}^{K}$ on $X$. We denote by $X_{-1, b}^{K}$ the analogue of the space $X_{-1}$ discussed earlier, for the operator $A_{b}^{K}$.

(2) There exists $H_{b} \in \mathcal{L}\left(Y, X_{-1, b}^{K}\right)$ that is an admissible control operator for $\mathbb{S}^{K}$, such that

$$
-A x=A_{b}^{K} x-H_{b} C x \quad \forall x \in \mathcal{D}(A) .
$$

For $(A, C)$ and $H_{b}$ as in Definition 3.1, $H_{b}$ is called a stabilizing backward output injection operator for $(A, C)$.

Note that in the particular situation when $\mathbb{T}$ is invertible (i.e., it can be extended to a group), $(A, C)$ is backward estimatable iff $(-A, C)$ is estimatable.

Proposition 3.2. Assume that $A$ is the generator of a strongly continuous group $\mathbb{T}$ on $X$, $C \in \mathcal{L}\left(X_{1}, Y\right)$ is an admissible observation operator for $\mathbb{T}$ and the pair $(A, C)$ is exactly observable. Then $(A, C)$ is forward and backward estimatable.

Proof. By Remark 2.3, $(A, C)$ is estimatable. On the other hand, since $A$ is the generator of a strongly continuous group $\mathbb{T}$, the exact observability of $(A, C)$ easily implies that $(-A, C)$ is exactly observable. Consequently, $(A, C)$ is also backward estimatable.

Proposition 3.3. With the notation and assumptions in Proposition 2.5, suppose that $(A, C)$ is backward estimatable and let $A_{b}^{K}, H_{b}$ be as in (3.1). Let $\tau>0$ and let $w_{b}$ be the solution, in a sense similar to (2.1), of the backward problem

$$
\left\{\begin{array}{l}
\dot{w}_{b}(t)=-A_{b}^{K} w_{b}(t)+H_{b} y(t) \text { for } t \leqslant \tau \\
w_{b}(\tau)=w(\tau)
\end{array}\right.
$$

where $w$ is the solution of (2.4). Then

$$
w_{b}(0)-z_{0}=\mathbb{S}_{\tau}^{K} \mathbb{T}_{\tau}^{K}\left(w_{0}-z_{0}\right),
$$

where $\mathbb{S}^{K}$ is the semigroup generated by $A_{b}^{K}$. Moreover, the pair $(A, C)$ is exactly observable in any time $\tau$ such that $\left\|\mathbb{S}_{\tau}^{K} \mathbb{T}_{\tau}^{K}\right\|<1$.

Proof. From the definition of $z$ (in Proposition 2.5),

$$
z(t)-z(\tau)=-\int_{t}^{\tau} A z(\xi) \mathrm{d} \xi \quad \forall t \in[0, \tau]
$$

The fact that $w_{b} \in C([0, \infty) ; X)$ satisfies (3.2) implies that for all $t \in[0, \tau]$,

$$
w_{b}(t)-w_{b}(\tau)=\int_{t}^{\tau} A_{b}^{K} w_{b}(\xi) \mathrm{d} \xi-\int_{t}^{\tau} H_{b} y(\xi) \mathrm{d} \xi
$$


For $t \geqslant 0$ we set $e_{b}(t)=w_{b}(t)-z(t)$. By subtracting side by side (3.4) from (3.5) and using the weak version of (3.1) it follows that

$$
e_{b}(t)-e_{b}(\tau)=\int_{t}^{\tau} A_{b}^{K} e_{b}(\xi) \mathrm{d} \xi \quad \forall t \in[0, \tau]
$$

which implies that

$$
e_{b}(t)=\mathbb{S}_{\tau-t}^{K} e_{b}(\tau) \quad \forall t \in[0, \tau] .
$$

Since $e_{b}(\tau)=w(\tau)-z(\tau)=\mathbb{T}_{\tau}^{K}\left(w_{0}-z_{0}\right)$ (see $\left.(2.5)\right)$, we obtain (3.3).

Finally we show that the pair $(A, C)$ is exactly observable in every time $\tau$ such that $\left\|\mathbb{S}_{\tau}^{K} \mathbb{T}_{\tau}^{K}\right\|<1$. First note that according to (3.3), if $w_{0}=0$ then

$$
w_{b}(0)=\left(I-\mathbb{S}_{\tau}^{K} \mathbb{T}_{\tau}^{K}\right) z_{0} .
$$

Since $\left\|\mathbb{S}_{\tau}^{K} \mathbb{T}_{\tau}^{K}\right\|<1$, the operator in the above bracket is invertible, so that

$$
z_{0}=\left(I-\mathbb{S}_{\tau}^{K} \mathbb{T}_{\tau}^{K}\right)^{-1} w_{b}(0) .
$$

Note that from (2.4) and (3.2),

$$
\left\{\begin{array}{l}
w(\tau)=-\int_{0}^{\tau} \mathbb{T}_{\tau-s}^{K} H y(s) \mathrm{d} s \\
w_{b}(0)=\mathbb{S}_{\tau}^{K} w(\tau)-\int_{0}^{\tau} \mathbb{S}_{s}^{K} H_{b} y(s) \mathrm{d} s .
\end{array}\right.
$$

Substituting the first formula into the second, it follows that

$$
w_{b}(0)=-\mathbb{S}_{\tau}^{K} \int_{0}^{\tau} \mathbb{T}_{\tau-s}^{K} H y(s) \mathrm{d} s-\int_{0}^{\tau} \mathbb{S}_{s}^{K} H_{b} y(s) \mathrm{d} s .
$$

Since $H$ and $H_{b}$ are admissible control operators for $\mathbb{T}^{K}$ and $\mathbb{S}^{K}$ respectively, it follows that there exists a constant $K_{\tau}>0$ such that

$$
\left\|w_{b}(0)\right\| \leqslant K_{\tau}\|y\|_{L^{2}([0, \tau], Y)} .
$$

This together with (3.6) implies the exact observability of $(A, C)$ in time $\tau$.

Remark 3.4. The above proposition can be seen as a generalization of the dual form of Russell's principle (see Russell [30], [29]) which asserts that for an operator group, forward and backward stabilizability implies exact controllability. In [8] an abstract version was given for systems with bounded input operators, and a more general abstract version was given in Rebarber and Weiss [27] (see the next remark for more detailed comments on this). All these references assume that the system is time-reversible.

Remark 3.5. Following the approach in [27], Proposition 3.3 could be generalized in the following way: for every $\alpha \in \mathbb{R}$, we define $\alpha$-estimatability of $(A, C)$ to mean that $(A-\alpha I, C)$ is estimatable. In this case there exists an operator $A^{K}: \mathcal{D}\left(A^{K}\right) \rightarrow X$ that generates a semigroup $\mathbb{T}^{K}$ on $X$ with growth bound $\omega\left(\mathbb{T}^{K}\right)<\alpha$, and there exists $H \in$ $\mathcal{L}\left(Y, X_{-1}^{K}\right)$ such that $(2.2)$ holds. (The proof of this is very easy.)

Backward $\alpha$-estimatability is defined similarly: $(A, C)$ is backward $\alpha$-estimatable if the pair $(A+\alpha I, C)$ is backward estimatable. In this case there exists an operator $A_{b}^{K}$ : 
$\mathcal{D}\left(A^{K}\right) \rightarrow X$ that generates a semigroup $\mathbb{S}^{K}$ on $X$ with growth bound $\omega\left(\mathbb{S}^{K}\right)<\alpha$, and there exists $H_{b} \in \mathcal{L}\left(Y, X_{-1}^{K}\right)$ such that (3.1) holds.

In Proposition 3.3 we could replace the estimatability requirement with $\alpha$-estimatability and the backward estimatability requirement with backward $\beta$-estimatability, as long as $\alpha+\beta \leqslant 0$ (the conclusions of the proposition remain unchanged).

The proof is a straightforward extension of the proof of Proposition 3.3. The above claim is a generalization of the dual result of [27, Theorem 2.1] (it is more general here because we do not require the semigroup $\mathbb{T}$ to be invertible, and we do not require $C$ to be admissible). We mention that in [27] there was a sign error in the definition of $\alpha$ optimizability, the dual concept of $\alpha$-estimatability: $A+\alpha I$ appeared in place of $A-\alpha I$. There was a similar sign error also in the definition of backward $\alpha$-optimizability.

In the particular case of time-reversible systems, we have the following:

Proposition 3.6. Let $A$ be the generator of a strongly continuous group $\mathbb{T}$ on $X$ and let $C \in \mathcal{L}\left(X_{1}, Y\right)$. Assume the following:

(a) $(A, C)$ is estimatable.

(b) The operators $\mathbb{T}_{t}$ are uniformly bounded for $t \leqslant 0$.

Then $(A, C)$ is exactly observable.

Proof. Let $\mathbb{T}^{K}$ be the exponentially stable semigroup as in Definition 2.1. We argue exactly as in the proof of Proposition 3.3, but with $H_{b}=0$, so that $A_{b}^{K}=-A$ and $\mathbb{S}_{t}^{K}=\mathbb{T}_{-t}$. We have $\left\|\mathbb{S}_{\tau}^{K} \mathbb{T}_{\tau}^{K}\right\|=\left\|\mathbb{T}_{-\tau} \mathbb{T}_{\tau}^{K}\right\|<1$ for $\tau>0$ large enough, so that (3.6) holds, and the proof is finished in the same way as for Proposition 3.3.

It follows from this proposition and Proposition 3.2 that for a skew-adjoint $A$ and an admissible $C$, forward and backward estimatability are equivalent. In the particular case of skew-adjoint generators and bounded $C$, we have the following:

Proposition 3.7. Assume that $A$ is skew-adjoint on $X$ and let $\mathbb{T}$ be the unitary group on $X$ generated by $A$. Let $C \in \mathcal{L}(X, Y)$. Then the following assertions are equivalent :

(i) $(A, C)$ is estimatable.

(ii) $(A, C)$ is backward estimatable.

(iii) $(A, C)$ is exactly observable.

(iv) $A-C^{*} C$ generates an exponentially stable group $\mathbb{T}^{K}$.

Moreover, if $(A, C)$ is exactly observable in time $\tau_{0}$, then the semigroup $\mathbb{T}^{K}$ defined in (iv) satisfies $\left\|\mathbb{T}_{\tau}^{K}\right\|<1$ for every $\tau \geqslant \tau_{0}$.

Proof. The equivalence of $(i)-(i v)$ is contained (in dual form) in Theorem 2.3 in Liu [25]. (The equivalence of $(i),(i i)$ and (iii) is also an easy consequence of Propositions 3.2 and 3.6.) The last statement is Lemma 2.2 in Ito, Ramdani and Tucsnak [17]. 
Remark 3.8. Proposition 3.3 implies, in particular, that there exist $M \geqslant 1, \omega>0$ (independent of $\tau$ ) such that

$$
\left\|w_{b}(0)-z_{0}\right\| \leqslant M e^{-\omega \tau}\left\|w_{0}-z_{0}\right\|
$$

so that for large $\tau, w_{b}(0)$ is a good approximation of the initial state $z_{0}$.

The process of computing $w(\tau)$ using an observer and then computing $w_{b}(0)$ using the backward observer (3.2), as described in Proposition 3.3, may be regarded as one estimation cycle, as described (under much simpler assumptions) in Section 1. In the sequel we discuss iterative repetitions of such estimation cycles.

We give an algorithm allowing the approximation of $z_{0}$ provided that $\tau$ is such that $\left\|\mathbb{S}_{\tau}^{K} \mathbb{T}_{\tau}^{K}\right\|<1$. More precisely, for $\tau>0$ and $z_{0} \in \mathcal{D}(A)$, we consider the sequences $\left(z^{(n)}\right)$ and $\left(z_{b}^{(n)}\right)$ in $C([0, \tau], X)$ defined as follows: For every $n \geqslant 0$, we define $z^{(n)}$ and $z_{b}^{(n)}$ as the solutions of

$$
\begin{gathered}
\left\{\begin{array}{l}
\dot{z}^{(n)}(t)=A^{K} z^{(n)}(t)-H y(t), \\
z^{(n)}(0)=z_{b}^{(n-1)}(0) \text { for } n \geqslant 1, \\
z^{(0)}(0)=w_{0},
\end{array}\right. \\
\left\{\begin{array}{l}
\dot{z}_{b}^{(n)}(t)=-A_{b}^{K} z_{b}^{(n)}(t)+H_{b} y(t), \\
z_{b}^{(n)}(\tau)=z^{(n)}(\tau) .
\end{array}\right.
\end{gathered}
$$

Here $w_{0} \in X$ is an arbitrary initial guess for $z_{0}$.

Proposition 3.9. With the notation and assumptions in Propositions 2.5, 3.3, and using the above notation, for every $n \in \mathbb{N}$ we have

$$
z_{b}^{(n)}(0)-z_{0}=\left(\mathbb{S}_{\tau}^{K} \mathbb{T}_{\tau}^{K}\right)^{n+1}\left(w_{0}-z_{0}\right) .
$$

In particular if $\tau$ is such that $\alpha=\left\|\mathbb{S}_{\tau}^{K} \mathbb{T}_{\tau}^{K}\right\|<1$, then

$$
\left\|z_{b}^{(n)}(0)-z_{0}\right\| \leqslant \alpha^{n+1}\left\|w_{0}-z_{0}\right\| \quad \forall n \in \mathbb{N} .
$$

Proof. The functions $z^{(n)}$ and $z_{b}^{(n)}$ satisfy the assumptions on $w$ and $w_{b}$ in Proposition 3.3. It follows from (3.3) that for every integer $n \geqslant 0$

$$
z_{b}^{(n+1)}(0)-z_{0}=\mathbb{S}_{\tau}^{K} \mathbb{T}_{\tau}^{K}\left(z^{(n)}(0)-z_{0}\right),
$$

The above formula clearly implies (3.9). The last conclusion is then straightforward.

Remark 3.10. If $w_{0}=0$, then the iterative algorithm for approximating $z_{0}$ given in the above proposition is equivalent to performing the inversion in (3.6) using the corresponding Neumann series. We leave it to the reader to verify this fact.

Remark 3.11. In the colocated case $A=-A^{*}$ and $H=H_{b}=-C^{*}$ (discussed in Remark 2.4) the approximation algorithm in Proposition 3.9 can be reformulated in an interesting alternative way such that the forward and backward problems (3.7) and (3.8) become forward problems with the same generator and with zero initial data. For this, first we 
introduce the time reflection operators $\boldsymbol{I}_{\tau}$ for every $\tau>0$, as follows: if $u$ is a function defined on the interval $[0, \tau]$, then $\left(\boldsymbol{\Omega}_{\tau} u\right)(t)=u(\tau-t)$. For $n \geqslant 0$ we define the sequences

$$
\left\{\begin{array}{l}
v^{(2 n)}=z^{(n+1)}-z_{b}^{(n)} \\
v^{(2 n+1)}=\boldsymbol{Я}_{\tau}\left(z_{b}^{(n+1)}-z^{(n+1)}\right) .
\end{array}\right.
$$

We clearly have

$$
v^{(n)}(0)=0 \quad \forall n \geqslant 0 .
$$

Moreover, it is not difficult to check that for every $n \geqslant 0$ we have

$$
z_{b}^{(n)}=z_{b}^{(0)}+\sum_{k=0}^{n-1}\left(v^{(2 k)}+\boldsymbol{Я}_{\tau} v^{(2 k+1)}\right) .
$$

In particular, by using the above relation at time $t=0$ and the initial condition (3.11), we obtain that

$$
z_{0}=\lim _{n \rightarrow \infty} z_{b}^{(n)}(0)=z_{b}^{(0)}(0)+\sum_{k=0}^{\infty} v^{(2 k+1)}(\tau) .
$$

From (3.7)-(3.8) it easily follows that

$$
\left\{\begin{array}{l}
\dot{v}^{(2 n)}=A^{K} v^{(2 n)}+2 C^{*}\left(y-C z_{b}^{(n)}\right), \\
\dot{v}^{(2 n+1)}=A^{K} v^{(2 n+1)}-2 \boldsymbol{Я}_{\tau} C^{*}\left(y-C z_{b}^{(n+1)}\right) .
\end{array}\right.
$$

The alternative algorithm consists of solving the above equations forward in time, using the homogeneous initial conditions (3.11). As $z_{b}^{(n)}$ can be expressed by (3.12) in terms of $v^{(k)}$ for $k \leqslant 2 n-1$, we can compute the sequences $v^{(2 n)}$ and $v^{(2 n+1)}$ recursively. We can obtain an approximation of $z_{0}$ by truncating the series in (3.13). The above alternative algorithm has been proposed in Phung and Zhang [26] in the case of a Kirchhoff plate equation with distributed observation, so that their $C$ is bounded from $X$ to $Y$.

\section{A class of second order systems with unbounded observation}

In this section we show that our main results can be applied to a class of second order systems with unbounded observation operators studied, for example, in Weiss and Tucsnak [39]. Most of the systems modeling the linear vibrations of elastic systems can be written in the form described below. Note that the class of systems discussed in this section are not time reversible, in general (see Subsection 5.2).

First we introduce some notation. Let $H$ be a Hilbert space, and let $A_{0}: \mathcal{D}\left(A_{0}\right) \rightarrow H$ be a strictly positive operator. We introduce the scale of Hilbert spaces $H_{\alpha}, \alpha \in \mathbb{R}$, as follows: for every $\alpha \geqslant 0, H_{\alpha}=\mathcal{D}\left(A_{0}^{\alpha}\right)$, with the norm $\|\varphi\|_{\alpha}=\left\|A_{0}^{\alpha} \varphi\right\|_{H}$. The space $H_{-\alpha}$ is defined by duality with respect to the pivot space $H$ as follows: $H_{-\alpha}=H_{\alpha}^{\prime}$ for $\alpha>0$. Equivalently, $H_{-\alpha}$ is the completion of $H$ with respect to the norm $\|\varphi\|_{-\alpha}=\left\|A_{0}^{-\alpha} \varphi\right\|_{H}$. The operator $A_{0}$ can be extended (or restricted) to each $H_{\alpha}$, such that it becomes a bounded operator

$$
A_{0}: H_{\alpha} \rightarrow H_{\alpha-1} \quad \forall \alpha \in \mathbb{R}
$$

Let $C_{0} \in \mathcal{L}\left(H_{\frac{1}{2}}, Y\right)$, where $Y$ is another Hilbert space. We identify $Y$ with its dual, so that $Y=Y^{\prime}$. We denote $B_{0}=C_{0}^{*}$, so that $B_{0} \in \mathcal{L}\left(Y, H_{-\frac{1}{2}}\right)$. 
Consider the system described by

$$
\begin{gathered}
\frac{\mathrm{d}^{2}}{\mathrm{~d} t^{2}} q(t)+A_{0} q(t)+\gamma B_{0} \frac{\mathrm{d}}{\mathrm{d} t} C_{0} q(t)=0, \\
y(t)=\frac{\mathrm{d}}{\mathrm{d} t} C_{0} q(t),
\end{gathered}
$$

where $\gamma \geqslant 0$ is a constant and $t \in[0, \infty)$ is the time. The equation (4.2) is understood as an equation in $H_{-\frac{1}{2}}$ (i.e., all the terms are in $H_{-\frac{1}{2}}$ ). Here, $q$ stands for the displacement field and the term $\gamma B_{0} \frac{\mathrm{d}}{\mathrm{d} t} C_{0} q(t)$, informally written as $\gamma B_{0} C_{0} \dot{q}(t)$, represents a viscous damping. The state $z(t)$ of this system and its state space $X$ are defined by

$$
z(t)=\left[\begin{array}{c}
q(t) \\
\dot{q}(t)
\end{array}\right], \quad X=H_{\frac{1}{2}} \times H
$$

It is easy to verify that the equations (4.2), (4.3) are equivalent to the following system of first order equations:

$$
\left\{\begin{array}{l}
\dot{z}(t)=A(\gamma) z(t) \\
y(t)=C z(t)
\end{array}\right.
$$

where

$$
\begin{gathered}
A(\gamma)=\left[\begin{array}{cc}
0 & I \\
-A_{0} & -\gamma B_{0} C_{0}
\end{array}\right], \\
\mathcal{D}(A(\gamma))=\left\{\left[\begin{array}{c}
\varphi \\
\psi
\end{array}\right] \in H_{\frac{1}{2}} \times H_{\frac{1}{2}} \mid A_{0} \varphi+\gamma B_{0} C_{0} \psi \in H\right\}, \\
C: \mathcal{D}(A(\gamma)) \rightarrow Y, \quad C=\left[\begin{array}{ll}
0 & C_{0}
\end{array}\right] .
\end{gathered}
$$

It is well known that $A(0)$ generates a unitary group in $X$ (see, for instance, [35, Proposition 3.8.7]). For every $\gamma>0$ the operator $A(\gamma)$ generates a semigroup of contractions (see, for instance, [39, Proposition 5.1]). It is not difficult to check that the adjoint of $A(\gamma)$ is given by

$$
\begin{gathered}
\mathcal{D}\left(A(\gamma)^{*}\right)=\left\{\left[\begin{array}{l}
\varphi \\
\psi
\end{array}\right] \in H_{\frac{1}{2}} \times H_{\frac{1}{2}} \mid A_{0} \varphi-\gamma B_{0} C_{0} \psi \in H\right\} \\
A(\gamma)^{*}=\left[\begin{array}{cc}
0 & -I \\
A_{0} & -\gamma B_{0} C_{0}
\end{array}\right] .
\end{gathered}
$$

The result below shows that the results in Section 2 can be applied to the system (4.4).

Proposition 4.1. With the above notation, assume that $A(1)$ is the generator of an exponentially stable semigroup $\mathbb{T}^{K}$ on $X$. Then for every $\gamma \geqslant 0$, the pair $(A(\gamma), C)$ is estimatable and backward estimatable. The corresponding output injection operators are

$$
H=\left[\begin{array}{c}
0 \\
(\gamma-1) B_{0}
\end{array}\right], \quad H_{b}=\left[\begin{array}{c}
0 \\
-(\gamma+1) B_{0}
\end{array}\right] .
$$

Proof. Let $X_{-1,1}$ be the dual of $\mathcal{D}\left(A(1)^{*}\right)$ with respect to the pivot space $X$. In this proof, inner products in $H$ or in $Y$ will not have subscripts, the others will. In order to show that $(A(\gamma), C)$ is estimatable, we prove below that the relation

$$
A(1) z=A(\gamma) z+H C z \quad \forall z \in \mathcal{D}(A(\gamma))
$$


holds in $X_{-1,1}$. Let $z=\left[\begin{array}{l}\varphi \\ \psi\end{array}\right] \in \mathcal{D}(A(\gamma))$ and $\phi=\left[\begin{array}{l}\xi \\ \eta\end{array}\right] \in \mathcal{D}\left((A(1))^{*}\right)$. Using (4.5), (4.6), (4.8) and (4.9) we get

$$
\begin{aligned}
\left\langle z,(A(1))^{*} \phi\right\rangle_{X}-\left\langle C z, H^{*} \phi\right\rangle & =\left\langle A_{0}^{\frac{1}{2}} \varphi, A_{0}^{\frac{1}{2}} \eta\right\rangle \\
& +\left\langle\psi, A_{0} \xi-B_{0} C_{0} \eta\right\rangle-(\gamma-1)\left\langle C_{0} \psi, C_{0} \eta\right\rangle \\
= & -\left\langle A_{0} \varphi, \eta\right\rangle_{H_{-\frac{1}{2}}, H_{\frac{1}{2}}}+\left\langle A_{0}^{\frac{1}{2}} \psi, A_{0}^{\frac{1}{2}} \xi\right\rangle \\
& -\left\langle C_{0} \psi, C_{0} \eta\right\rangle-(\gamma-1)\left\langle C_{0} \psi, C_{0} \eta\right\rangle \\
& =\left\langle A_{0}^{\frac{1}{2}} \psi, A_{0}^{\frac{1}{2}} \xi\right\rangle-\left\langle A_{0} \varphi, \eta\right\rangle_{H_{-\frac{1}{2}}, H_{\frac{1}{2}}}-\gamma\left\langle B_{0} C_{0} \psi, \eta\right\rangle_{H_{-\frac{1}{2}}, H_{\frac{1}{2}}} .
\end{aligned}
$$

Since $z \in \mathcal{D}(A(\gamma))$, it follows that

$$
\left\langle z,(A(1))^{*} \phi\right\rangle_{X}-\left\langle C z, H^{*} \phi\right\rangle=\left\langle A_{0}^{\frac{1}{2}} \psi, A_{0}^{\frac{1}{2}} \xi\right\rangle \quad-\left\langle A_{0} \varphi+\gamma B_{0} C_{0} \psi, \eta\right\rangle=\langle A(\gamma) z, \phi\rangle_{X} .
$$

According to Definition 2.1, the above formula implies that the pair $(A(\gamma), C)$ is estimatable with the output injection operator $H$ given by (4.10).

Let $X_{-1,1}^{d}$ be the dual of $\mathcal{D}(A(1))$ with respect to the pivot space $X$. In order to show that $(A(\gamma), C)$ is backward estimatable we prove below that the relation

$$
A(1)^{*} z=-A(\gamma) z+H_{b} C z \quad \forall z \in \mathcal{D}(A(\gamma))
$$

holds in $X_{-1,1}^{d}$. Let $z=\left[\begin{array}{l}\varphi \\ \psi\end{array}\right] \in \mathcal{D}(A(\gamma))$ and $\phi=\left[\begin{array}{l}\xi \\ \eta\end{array}\right] \in \mathcal{D}(A(1))$. Using (4.5), (4.6), (4.8) and (4.9), we get

$$
\begin{aligned}
\langle z, A(1) \phi\rangle_{X}-\left\langle C z, H_{b}^{*} \phi\right\rangle & =\left\langle A_{0}^{\frac{1}{2}} \varphi, A_{0}^{\frac{1}{2}} \eta\right\rangle \\
& -\left\langle\psi, A_{0} \xi+B_{0} C_{0} \eta\right\rangle+(\gamma+1)\left\langle C_{0} \psi, C_{0} \eta\right\rangle \\
= & \left\langle A_{0} \varphi, \eta\right\rangle_{H_{-\frac{1}{2}}, H_{\frac{1}{2}}}-\left\langle A_{0}^{\frac{1}{2}} \psi, A_{0}^{\frac{1}{2}} \xi\right\rangle \\
& -\left\langle C_{0} \psi, C_{0} \eta\right\rangle+(\gamma+1)\left\langle C_{0} \psi, C_{0} \eta\right\rangle \\
= & \left\langle A_{0}^{\frac{1}{2}}(-\psi), A_{0}^{\frac{1}{2}} \xi\right\rangle+\left\langle A_{0} \varphi, \eta\right\rangle_{H_{-\frac{1}{2}}, H_{\frac{1}{2}}} \\
& +\gamma\left\langle B_{0} C_{0} \psi, \eta\right\rangle_{H_{-\frac{1}{2}}, H_{\frac{1}{2}}}
\end{aligned}
$$

Since $z \in \mathcal{D}(A(\gamma))$, it follows that

$$
\begin{aligned}
\left\langle z,(A(1))^{*} \phi\right\rangle_{X}-\left\langle C z, H_{b}^{*} \phi\right\rangle= & \left\langle A_{0}^{\frac{1}{2}}(-\psi), A_{0}^{\frac{1}{2}} \xi\right\rangle \\
& +\left\langle A_{0} \varphi+\gamma B_{0} C_{0} \psi, \eta\right\rangle=-\langle A(\gamma) z, \phi\rangle_{X} .
\end{aligned}
$$

According to Definition 2.1, the above formula implies that the pair $(-A(\gamma), C)$ is estimatable, with the output injection operator $H_{b}$ given by (4.10).

By combining the last result and Proposition 3.9 we obtain an algorithm to estimate the initial state of the system (4.2)-(4.3). More precisely, for every $n \geqslant 0$ we define the 
sequences $\left(q^{(n)}\right)$ and $\left(q_{b}^{(n)}\right)$ by

$$
\begin{aligned}
& \left\{\begin{array}{l}
\ddot{q}^{(n)}(t)+A_{0} q^{(n)}(t)+B_{0} C_{0} \dot{q}^{(n)}(t)=(1-\gamma) B_{0} y, \\
q^{(n)}(0)=q_{b}^{(n-1)}(0) \text { for } n \geqslant 1, \quad q_{b}^{(0)}(0)=w_{0}, \\
\dot{q}^{(n)}(0)=\dot{q}_{b}^{(n-1)}(0) \text { for } n \geqslant 1, \quad \dot{q}_{b}^{(0)}(0)=w_{1},
\end{array}\right. \\
& \left\{\begin{array}{l}
\ddot{q}_{b}^{(n)}(t)+A_{0} q_{b}^{(n)}(t)-B_{0} C_{0} \dot{q}_{b}^{(n)}(t)=-(1+\gamma) B_{0} y, \\
q_{b}^{(n)}(\tau)=q^{(n)}(\tau), \\
\dot{q}_{b}^{(n)}(\tau)=\dot{q}^{(n)}(\tau) .
\end{array}\right.
\end{aligned}
$$

Here $w_{0} \in H_{\frac{1}{2}}$ and $w_{1} \in H$ are arbitrary initial guesses for $q_{0}$ and $q_{1}$.

Recall from Proposition 4.1 that $\mathbb{T}^{K}$ denotes the semigroup generated by $A(1)$. From Proposition 3.9 we obtain the following:

Corollary 4.2. Assume that $\mathbb{T}^{K}$ is exponentially stable and let $\tau>0$ be such that $\left\|\mathbb{T}_{\tau}^{K}\right\|<$ 1. Denote $\alpha=\left\|\mathbb{T}_{\tau}^{K}\right\|^{4}$. Then for all $n \in \mathbb{N}$,

$$
\begin{aligned}
\left\|q_{b}^{(n)}(0)-q(0)\right\|_{\frac{1}{2}}^{2}+\left\|\dot{q}_{b}^{(n)}(0)-\dot{q}(0)\right\|_{H}^{2} & \\
& \leqslant \alpha^{n+1}\left(\left\|w_{0}-q(0)\right\|_{\frac{1}{2}}^{2}+\left\|w_{1}-\dot{q}(0)\right\|_{H}^{2}\right) .
\end{aligned}
$$

The second example in Section 5 shows that the above abstract setting can be applied to a system described by the wave equation in a domain $\Omega \subset \mathbb{R}^{n}$ with velocity observation on a part of the boundary.

\section{$5 \quad$ Examples and numerical results}

In this section we apply our algorithm to reconstruct the initial state for the Schrödinger equation and the wave equation in the unit square $\Omega \subset \mathbb{R}^{2}$. In the first case, the observation is distributed on a subset of $\Omega$, whereas in the second case the observation is localized on two adjacent sides of $\Omega$.

All the computations were performed on a Power Mac G4 $2.8 \mathrm{GHz}$ with 8 Go DDR SDRM and the algorithms were developed under Matlab.

\subsection{The Schrödinger equation}

In this subsection we consider the problem of estimating the initial state of a system described by the Schrödinger equation with observation on a part of the domain.

Let $\Omega$ denote a rectangular domain in $\mathbb{R}^{d}, d \geqslant 1$. We consider the following initial and boundary value problem:

$$
\left\{\begin{aligned}
\dot{z}+i \Delta z & =0, & & x \in \Omega, \quad t \in[0,+\infty) \\
z & =0, & & x \in \partial \Omega, t \in[0,+\infty) \\
z(x, 0) & =z_{0}(x), & & x \in \Omega .
\end{aligned}\right.
$$


Given a nonempty open subset $\mathcal{O} \subset \Omega$, we consider the output

$$
y=z_{\mid \mathcal{O}}
$$

Equations (5.1)-(5.2) can be written in the standard form

$$
\dot{z}=A z, \quad y=C z,
$$

if we introduce the following spaces and operators:

- the state space is $X=L^{2}(\Omega)$,

- $A: \mathcal{D}(A)=\mathcal{H}^{2}(\Omega) \cap \mathcal{H}_{0}^{1}(\Omega) \rightarrow X$ is the skew-adjoint operator defined by

$$
A \varphi=-i \Delta \varphi \quad \forall \varphi \in \mathcal{D}(A) .
$$

- the output space is $Y=L^{2}(\mathcal{O})$,

- the observation operator $C \in \mathcal{L}(X, Y)$ is defined by

$$
C \varphi=\varphi_{\mid \mathcal{O}} \quad \forall \varphi \in X .
$$

According to Komornik [18] the pair $(A, C)$ is exactly observable in any time $\tau>0$. According to Proposition $3.7(A, C)$ is estimatable and backward estimatable. Clearly the same statements hold also for $(-A, C)$. Moreover, the semigroups $\mathbb{T}^{K}$ and $\mathbb{S}^{K}$ generated by $A-C^{*} C$ and $-A-C^{*} C$, respectively, satisfy $\left\|\mathbb{T}_{\tau}^{K}\right\|<1$ and $\left\|\mathbb{S}_{\tau}^{K}\right\|<1$ for every $\tau>0$.

According to Proposition 3.9 the initial state of the system (5.1) can be approximated using the output data (5.2) by the recursive algorithm

$$
\begin{aligned}
& \left\{\begin{array}{l}
\dot{z}^{(n)}(t)=\left(-i \Delta-\chi_{\mathcal{O}}\right) z^{(n)}(t)+y(t), \\
z^{(n)}(0)=z_{b}^{(n-1)}(0) \text { for } n \geqslant 1, \\
z^{(0)}(0)=w_{0},
\end{array}\right. \\
& \left\{\begin{array}{l}
\dot{z}_{b}^{(n)}(t)=\left(-i \Delta+\chi_{\mathcal{O}}\right) z_{b}^{(n)}(t)-y(t), \\
z_{b}^{(n)}(\tau)=z^{(n)}(\tau) .
\end{array}\right.
\end{aligned}
$$

In the above equations we have suppressed the dependence on the position $x . \chi_{\mathcal{O}}$ is the characteristic function of $\mathcal{O}$ and $y$ is extended by zero outside $\mathcal{O}$. As usual, $w_{0} \in X$ is an arbitrary initial guess for $z_{0}$.

In order to show the efficiency of our iterative algorithm, we consider the particular case where $\Omega$ is the unit square in $\mathbb{R}^{2}, \mathcal{O}=(1 / 3,2 / 3) \times(1 / 3,2 / 3), \tau=0.2$ (note that $\tau$ can be chosen very small, as $(A, C)$ is exactly observable in arbitrarily small time) and the initial data to be recovered is $z_{0}\left(x_{1}, x_{2}\right)=4 x\left(1-x_{1}\right) \sin \left(5 \pi x_{2}\right)+i \sin \left(3 \pi x_{1}\right) \sin \left(2 \pi x_{2}\right)$. We use a Crank-Nicolson scheme in time combined to a finite difference space discretization to simulate the forward and backward systems (5.5) and (5.6). The initial guess $w_{0}$ is taken to be zero, the time step is $\Delta t=\tau / 300$ and the number of space discretization points is $N_{x_{1}}=N_{x_{2}}=150$ in each direction. Figure 1 shows the evolution of the relative error (in \%) as a function of the number of iterations for a noisy observation with a noise level of $10 \%$ (we used a noise obtained by adding random numbers uniformly distributed in $\left[-\|y\|_{L^{\infty}} / 10,\|y\|_{L^{\infty}} / 10\right]$ to all entries of $\left.y(t)\right)$. After 10 iterations, the relative error decreases from $19.7 \%$ to $0.5 \%$. The error can be further reduced either by reducing the mesh sizes or by using high-order solvers. Each forward or backward iteration of the algorithm required approximately 20 seconds. 


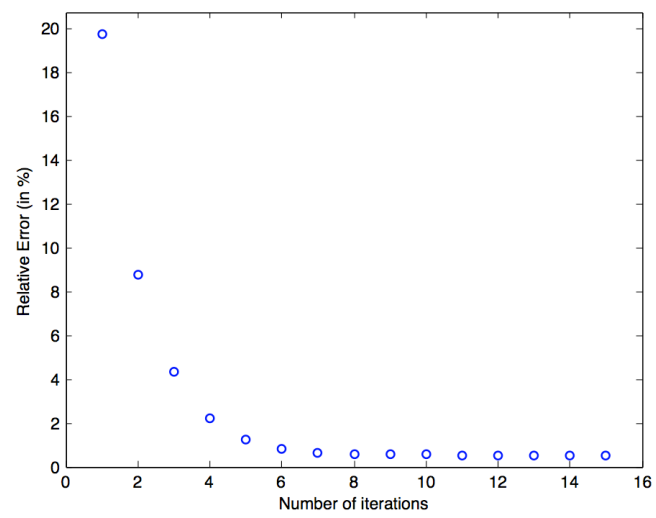

Figure 1: Relative error (in \%) as a function of the number of iterations.

\subsection{The wave equation}

In this subsection we consider the problem of estimating the initial state of a system described by the wave equation with velocity observation on a part of the boundary.

Let $\Omega \subset \mathbb{R}^{d}$ be a bounded domain with boundary $\Gamma$ and let $\Gamma_{0}$ and $\Gamma_{1}$ be subsets of $\Gamma$. Assume that one of the two following assumptions holds:

- (H1): $\Gamma$ is of class $C^{\infty}, \Gamma_{0}$ and $\Gamma_{1}$ are relatively open (and also relatively closed) and nonempty subsets of $\Gamma$ such that $\Gamma_{0} \cap \Gamma_{1}=\emptyset$ and $\Gamma_{0} \cup \Gamma_{1}=\Gamma$. Moreover, $\Gamma_{1}$ satisfies the geometric optics condition of Bardos, Lebeau and Rauch [6].

- $(\mathrm{H} 2): \Omega=[0,1]^{2}, \Gamma_{0}=(\{1\} \times[0,1]) \cup([0,1] \times\{1\})$ and $\Gamma_{1}=\Gamma \backslash \Gamma_{0}$.

We denote by $x$ the space variable $(x \in \bar{\Omega})$. Consider the system

$$
\begin{cases}\ddot{q}(x, t)=\Delta q(x, t) & \text { in } \Omega \times[0, \infty), \\ q(x, t)=0 & \text { on } \Gamma_{0} \times[0, \infty), \\ \frac{\partial q}{\partial \nu}(x, t)+\gamma \dot{q}(x, t)=0 & \text { on } \Gamma_{1} \times[0, \infty), \\ q(x, 0)=q_{0}(x) & \text { in } \Omega, \\ \dot{q}(x, 0)=q_{1}(x) & \text { in } \Omega, \\ y(x, t)=\dot{q}(x, t) & \text { on } \Gamma_{1} \times[0, \infty),\end{cases}
$$

where $\gamma \geqslant 0$ is constant, $\nu$ is the unit outward normal to $\partial \Omega, y$ is the output function and the functions $q_{0}$ and $q_{1}$ are the initial state of the system. Note that the above system is not necessarily time-reversible. The easiest counterexample can be found by taking $\Omega$ one-dimensional and $\gamma=1$ (see, for instance, the last paragraph in [39]). Let us also note that the observation operator corresponding to (5.7) is not admissible (see, for instance, [5, p. 220] and [33]).

As shown, for instance, in [39], the above system can be written in the form (4.2), (4.3) with the following choice of spaces and operators:

$$
H=L^{2}(\Omega), \quad Y=L^{2}\left(\Gamma_{1}\right),
$$




$$
\begin{gathered}
H_{1}=\left\{f \in \mathcal{H}_{\Gamma_{0}}^{1}(\Omega)\left|\Delta f \in L^{2}(\Omega), \frac{\partial f}{\partial \nu}\right|_{\Gamma_{1}}=0\right\} \\
A_{0} \varphi=-\Delta \varphi \quad \forall \varphi \in \mathcal{D}\left(A_{0}\right)=H_{1}
\end{gathered}
$$

where $\mathcal{H}_{\Gamma_{0}}^{1}(\Omega)=\left\{f \in \mathcal{H}^{1}(\Omega) \mid f_{\mid \Gamma_{0}}=0\right\}$.

It is well-known that $A_{0}$ is self-adjoint, positive and boundedly invertible, see, for instance, Rodriguez-Bernal and Zuazua [28]. Moreover, $H_{\frac{1}{2}}=\mathcal{H}_{\Gamma_{0}}^{1}(\Omega)$.

The operator $C_{0} \in \mathcal{L}\left(H_{\frac{1}{2}}, Y\right)$ is defined by

$$
C_{0} f=f_{\mid \Gamma_{1}} \quad \forall f \in H_{\frac{1}{2}} .
$$

In order to determine $B_{0}=C_{0}^{*}$ we consider the operator $N \in \mathcal{L}\left(Y, H_{\frac{1}{2}}\right)$, called the Neumann map, with the following property: $N v=g$ if and only if $g \in \mathcal{H}_{\Gamma_{0}}^{\frac{1}{2}}(\Omega), \Delta g=0$ and $\frac{\partial g}{\partial \nu}=v$ on $\Gamma_{1}$. As shown in $[39$, Section 7$]$, we have $B_{0} \in \mathcal{L}\left(U, H_{-\frac{1}{2}}\right)$ and

$$
B_{0}=C_{0}^{*}=A_{0} N
$$

In order to determine the initial state $\left(q_{0}, q_{1}\right)$ of $(5.7)$ from the boundary observations $y$, we consider the sequences $\left(q^{(n)}\right)_{n \in \mathbb{N}}$ and $\left(q_{b}^{(n)}\right)_{n \in \mathbb{N}}$ defined by

$$
\begin{aligned}
& \left\{\begin{array}{l}
\ddot{q}^{(n)}(x, t)=\Delta q^{(n)}(x, t) \text { in } \Omega \times[0, \infty), \\
q^{(n)}(x, t)=0 \text { on } \Gamma_{0} \times[0, \infty), \\
\frac{\partial q^{(n)}}{\partial \nu}(x, t)+\dot{q}^{(n)}(x, t)=(1-\gamma) y(x, t) \text { on } \Gamma_{1} \times[0, \infty), \\
q^{(n)}(x, 0)=q_{b}^{(n-1)}(x, 0) \text { for } n \geqslant 1 \text { in } \Omega, \\
\dot{q}^{(n)}(x, 0)=\dot{q}_{b}^{(n-1)}(x, 0) \text { for } n \geqslant 1 \text { in } \Omega, \\
q^{(0)}(x, 0)=w_{0}(x) \text { in } \Omega, \\
\dot{q}^{(0)}(x, 0)=w_{1}(x) \text { in } \Omega,
\end{array}\right. \\
& \left\{\begin{array}{l}
\ddot{q}_{b}^{(n)}(x, t)=\Delta q_{b}^{(n)}(x, t) \text { in } \Omega \times[0, \infty), \\
q_{b}^{(n)}(x, t)=0 \text { on } \Gamma_{0} \times[0, \infty), \\
\frac{\partial q_{b}^{(n)}}{\partial \nu}(x, t)-\dot{q}_{b}^{(n)}(x, t)=-(1+\gamma) y(x, t) \text { on } \Gamma_{1} \times[0, \infty), \\
q_{b}^{(n)}(x, \tau)=q^{(n)}(x, \tau) \text { in } \Omega, \\
\dot{q}_{b}^{(n)}(x, \tau)=\dot{q}^{(n)}(x, \tau) \text { in } \Omega .
\end{array}\right.
\end{aligned}
$$

As usual, $w_{0} \in H_{\frac{1}{2}}$ and $w_{1} \in H$ are arbitrary initial guesses for $q_{0}$ and $q_{1}$.

These equations are obtained by specializing to the above choice of $A_{0}$ and $C_{0}$ the iterative scheme from (4.11) and (4.12). From Corollary 4.2 we obtain:

Corollary 5.1. If $q_{0} \in H_{1}, q_{1} \in H_{\frac{1}{2}}$ and one of the assumptions (H1) or (H2) holds, then for $\tau$ sufficiently large, there exists $\alpha \in(0,1)$ such that, for all $n \in \mathbb{N}$,

$$
\begin{aligned}
\left\|\nabla q_{b}^{(n)}(\cdot, 0)-\nabla q_{0}\right\|_{L^{2}(\Omega)}^{2}+\| \dot{q}_{b}^{(n)}(\cdot, 0) & -q_{1} \|_{L^{2}(\Omega)}^{2} \\
& \leqslant \alpha^{n+1}\left(\left\|\nabla w_{0}-\nabla q_{0}\right\|_{L^{2}(\Omega)}^{2}+\left\|w_{1}-q_{1}\right\|_{L^{2}(\Omega)}^{2}\right) .
\end{aligned}
$$


Proof. If $(H 1)$ holds, the geometric optics condition insures the exponential stability of (5.7) with $\gamma=1$, see [6]. If (H2) holds, the exponential stability of (5.7) with $\gamma=1$ follows from Cornilleau et al. [9, Remark 2].

Concerning the minimal time required in the above corollary, we conjecture that it is given by the maximal time needed by a ray of geometric optics before intersecting $\Gamma_{1}$. In particular, if $(H 2)$ holds, this time should be $2 \sqrt{2}$. Nevertheless, there exist many initial states which can be recovered from data from a shorter observation time.

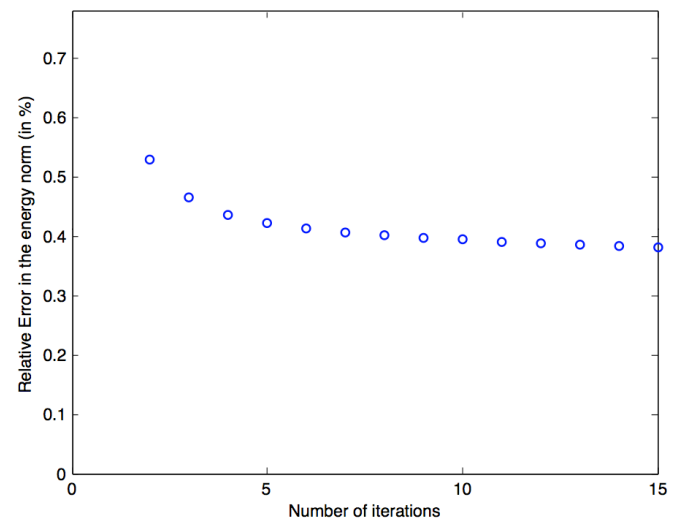

Figure 2: Relative error (in \%) on $\left(q_{0}, q_{1}\right)$ for the norm in $X=H_{\frac{1}{2}} \times H$ as a function of the number of iterations.

As a numerical illustration of our iterative algorithm, we consider problem (5.7) on the unit square $\Omega=[0,1]^{2}$ and with $\gamma=1$ (non reversible problem). The initial data to be recovered are given by the "bubble" function $q_{0}(x)=q_{1}(x)=x(1-x) y(1-y)$ and the time of observation is $\tau=2 \sqrt{2}$ which is the exact observability time of the problem. To solve numerically (5.9) and (5.10), we use an explicit centered scheme in time combined to $\mathbb{P}^{1}$-finite elements for the space discretization on a structured mesh. The initial guess $\left(w_{0}, w_{1}\right)$ in $(5.9)$ is chosen to be zero (and thus the initial relative error is $100 \%$ ) and we used a noisy observation (random noise with level $10 \%$ obtained by adding random numbers uniformly distributed in $\left[-\|y\|_{L^{\infty}} / 10,\|y\|_{L^{\infty}} / 10\right]$ to all entries of $\left.y(t)\right)$. We used 35 points of space discretization in each direction and the time step $\Delta t=3 e-5$. Each forward or backward iteration of the algorithm requires approximatively 30 seconds.

Figure 2 shows the decay of the relative error in the energy norm (i.e. for the norm in $\left.X=H_{\frac{1}{2}} \times H\right)$ : after 15 iterations, this error is about $0.4 \%$.

Figure 3 shows the relative errors between the exact and estimated initial data, separately for $q_{0}$ and $q_{1}$. After 15 iterations, the relative error obtained is about $0.27 \%$ for the approximation of $q_{0}$ and about $1.2 \%$ for $q_{1}$. As we used a low order solver, a large number of iterations is needed to obtain an accurate approximation of the initial time derivative $q_{1}$, while the second iteration provides a very precise approximation of $q_{0}$. Theses results can be improved by using high order schemes. 

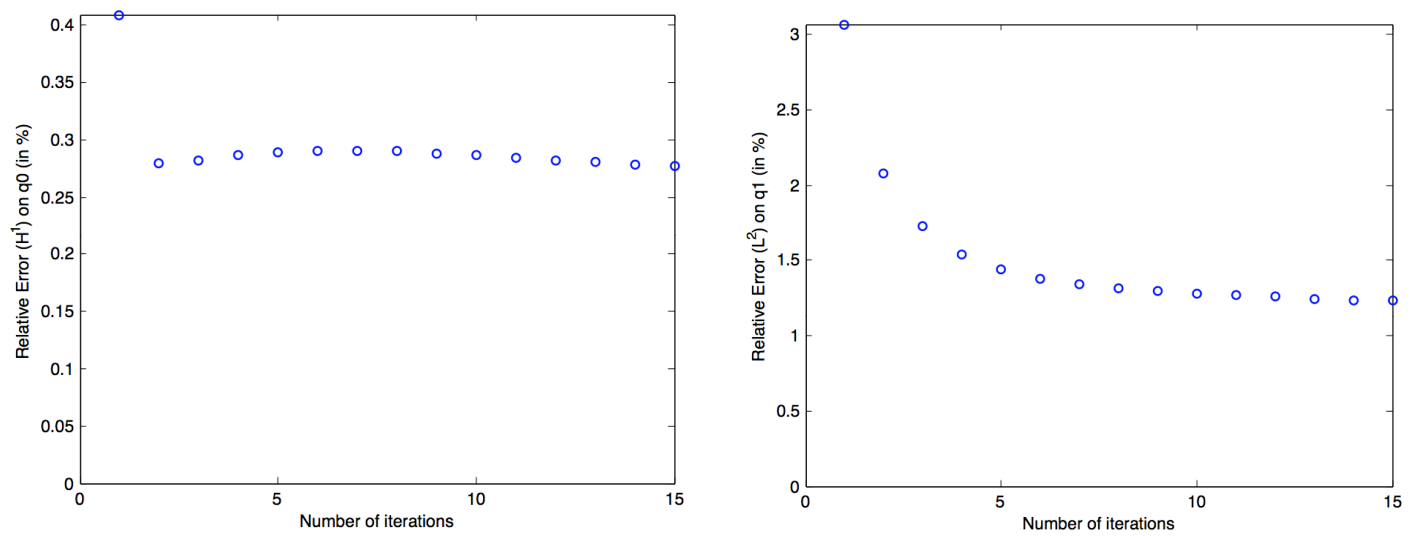

Figure 3: Top: The norm in $H_{\frac{1}{2}}$ of the relative error in $q_{0}$ (in $\%$ ) versus the number of iterations. Bottom: The norm in $H$ of the relative error on $q_{1}$ (in \%) versus the number of iterations.

\section{References}

[1] C. Alves, A. L. Silvestre, T. Takahashi, and M. Tucsnak. Solving inverse source problems using observability. applications to the Euler-Bernoulli plate equation. SIAM J. Control Optim., 48(3):1632-1659, 2009.

[2] K. Ammari and M. Tucsnak. Stabilization of second order evolution equations by a class of unbounded feedbacks. ESAIM Control Optim. Calc. Var., 6:361-386 (electronic), 2001.

[3] D. Auroux and J. Blum. Back and forth nudging algorithm for data assimilation problems. C. R. Acad. Sci. Paris Sér. I Math., 340:873-878, 2005.

[4] D. Auroux and J. Blum. A nudging-based data assimilation method: the back and forth nudging (bfn) algorithm. Nonlin. Proc. Geophys., 15(305-319), 2008.

[5] S. A. Avdonin and S. A Ivanov. Families of exponentials - The method of moments in controllability problems for distributed parameter systems. Cambridge University Press, Cambridge, 1995.

[6] C. Bardos, G. Lebeau, and J. Rauch. Sharp sufficient conditions for the observation, control and stabilization of waves from the boundary. SIAM J. Control Optim., 30:1024-1065, 1992.

[7] S. Bonnabel, P. Martin, and P. Rouchon. Symmetry-preserving observers. IEEE Trans. Automat. Control, 53(11):2514-2526, 2008.

[8] G. Chen. Control and stabilization for the wave equation in a bounded domain. SIAM J. Control Optim., 17:66-81, 1979.

[9] P. Cornilleau, J.-P. Lohéac, and A. Osses. Nonlinear neumann boundary stabilization of the wave equation using rotated multipliers. J. Dynamic. Control. Sys., To appear.

[10] R. F. Curtain and G. Weiss. Exponential stabilization of well-posed systems by colocated feedback. SIAM J. Control Optim., 45(1):273-297, 2006. 
[11] R. F. Curtain and H. Zwart. An introduction to infinite-dimensional linear systems theory, volume 21 of Texts in Applied Mathematics. Springer-Verlag, New York, 1995.

[12] J. Deguenon, G. Sallet, and C.-Z. Xu. Infinite dimensional observers for vibrating systems. In Proc. of IEEE Conf. on Decision and Control, pages 3979-3983, 2006.

[13] M. Fink, D. Cassereau, A. Derode, C. Prada, P. Roux, M. Tanter, J.-L. Thomas, and F. Wu. Time-reversed acoustics. Rep. Prog. Phys., 63(12):1933-1995, 2000.

[14] B. Gebauer and O. Scherzer. Impedance-acoustic tomography. SIAM J. Appl. Math., 69(2):565-576, 2008.

[15] B.-Z. Guo and Z.-C. Shao. Stabilization of an abstract second order system with application to wave equations under non-collocated control and observations. Syst. Control Lett., 58(5):334-341, 2009.

[16] A. Haraux. Une remarque sur la stabilisation de certains systèmes du deuxième ordre en temps. Portugal. Math., 46:245-258, 1989.

[17] K. Ito, K. Ramdani, and M. Tucsnak. A time reversal based algorithm for solving initial data inverse problems. Discrete Contin. Dyn. Syst. Ser. S, to appear.

[18] V. Komornik. On the exact internal controllability of a petrowsky system. J. Math. Pures Appl., 71:331-342, 1992.

[19] V. Komornik. Rapid boundary stabilization of linear distributed systems. SIAM J. Control Optim., 35:1591-1613, 1997.

[20] M. Krstic, B.-Z Guo, A. Balogh, and A. Smyshlyaev. Control of a tip-force destabilized shear beam by observer-based boundary feedback. SIAM J. Control Optim., 47(2):553-574, 2008.

[21] M. Krstic, L. Magnis, and R. Vazquez. Nonlinear control of the viscous burgers equation: Trajectory generation, tracking, and observer design. J. Dyn. Sys., Meas., Control, 131, 2009.

[22] P. Kuchment and L. Kunyansky. Mathematics of thermoacoustic tomography. European J. Appl. Math., 19(2):191-224, 2008.

[23] I. Lasiecka. Galerkin approximations of infinite-dimensional compensators for flexible structures with unbounded control action. Acta Appl. Math., 28:101-133, 1992.

[24] F.-X. Le Dimet, V. Shutyaev, and I. Gejadze. On optimal solution error in variational data assimilation: theoretical aspects. Russian J. Numer. Anal. Math. Modelling, 21(2):139-152, 2006.

[25] K. Liu. Locally distributed control and damping for the conservative systems. SIAM J. Control Optim., 35(5):1574-1590, 1997.

[26] K. D. Phung and X. Zhang. Time reversal focusing of the initial state for kirchhoff plate. SIAM J. Appl. Math., 68(6):1535-1556, 2008.

[27] R. Rebarber and G. Weiss. An extension of russell's principle on exact controllability. In Proceedings of the Fourth ECC (Brussels), 1997. 
[28] A. Rodriguez-Bernal and Enrique Zuazua. Parabolic singular limit of a wave equation with localized boundary damping. Discrete and Continuous Dynamical Systems, 1:303-346, 1995.

[29] D. L. Russell. Exact boundary value controllability theorems for wave and heat processes in star-complemented regions. In Differential games and control theory (Proc. NSF-CBMS Regional Res. Conf., Univ. Rhode Island, Kingston, R.I., 1973), pages 291-319. Lecture Notes in Pure Appl. Math., Vol. 10. Dekker, New York, 1974.

[30] D. L. Russell. Controllability and stabilizability theory for linear partial differential equations: recent progress and open questions. SIAM Rev., 20(4):639-739, 1978.

[31] M. Slemrod. A note on complete controllability and stabilizability for linear control systems in hilbert space. SIAM J. Control Optim., 12:500-508, 1974.

[32] A. Smyshlyaev and M. Krstic. Adaptive boundary control for unstable parabolic PDEs. II. Estimation-based designs. Automatica, 43(9):1543-1556, 2007.

[33] D. Tataru. On the regularity of boundary traces for the wave equation. Ann. Scuola Norm. Sup. Pisa Cl. Sci. (4), 26(1):185-206, 1998.

[34] J.J. Teng, G. Zhang, and S.X. Huang. Some theoretical problems on variational data assimilation. Appl. Math. Mech., 28(5):581-591, 2007.

[35] M. Tucsnak and G. Weiss. Observation and Control for Operator Semigroups. Birkäuser Advanced Texts. Birkäuser, Basel, 2009.

[36] J. M. Urquiza. Rapid exponential feedback stabilization with unbounded control operators. SIAM J. Control Optim., 43:2233-2244, 2005.

[37] G. Weiss and R. F. Curtain. Dynamic stabilization of regular linear systems. IEEE Trans. Automat. Control, 42:4-21, 1997.

[38] G. Weiss and R. Rebarber. Optimizability and estimatability for infinite-dimensional linear systems. SIAM J. Control Optim., 39(4):1204-1232, 2000.

[39] G. Weiss and M. Tucsnak. How to get a conservative well-posed linear system out of thin air. I. Well-posedness and energy balance. ESAIM Control Optim. Calc. Var., 9:247-274 (electronic), 2003.

[40] X. Zou, I.-M. Navon, and F.-X. Le Dimet. An optimal nudging data assimilation scheme using parameter estimation. Quart. J. Roy. Met. Soc., 118:1193-1186, 1992. 